\title{
Polarimeter and Spin Coherence Time Developments at COSY for a Storage Ring EDM Search
}

\author{
E. J. Stephenson ${ }^{1}$ \\ Center for Exploration of Energy and Matter \\ Indiana University, 2401 Milo. B. Sampson Lane, Bloomington, IN 47408 USA \\ E-mail: stephene@indiana.edu
}

\section{J. G. Onderwater}

Kernfysisch Versneller Instituut

University of Groningen, Zernikelaan 25

NL-9747 AA Groningen, The Netherlands

E-mail: onderwater@kvi.nI

\section{P. Lenisa}

University of Ferrara and INFN Ferrara

44100 Ferrara, Italy

E-mail: lenisalfe.infn.it

The Cooler Synchrotron (COSY) located at the Forschungszentrum Jülich along with the EDDA scintillation detectors have been used with a $0.97-\mathrm{GeV} / c$ polarized deuteron beam to demonstrate the feasibility of high efficiency polarimetry, correction of systematic errors, and long spin coherence times in preparation for the design and construction of a new storage ring to search for an electric dipole moment on the proton, deuteron, or other light nuclei. Slow extraction of the stored beam onto a $1.5-\mathrm{cm}$ thick carbon target yielded an efficiency (usefully detected particles divided by particles lost from the beam) near $10^{-3}$ and average $3 A_{y} / 2=$ 0.67(4). Calibration of the polarimeter's sensitivity to geometric and rate-induced systematic errors made possible the real time correction of such errors based on combinations of the primary detector rates to the level of $10^{-5}$ with the prospect for lower errors in the EDM search. Recently, new studies of manipulations of the stored beam polarization using an RF solenoid operating on the $1-G \gamma$ resonance demonstrated a spin coherence lifetime of minutes for a horizontally-polarized and electron-cooled beam without a Siberian Snake. Preparations are underway for examining the use of sextupole fields in the ring to lengthen this lifetime.

8th International Conference on Nuclear Physics at Storage Rings (Stori11)

Laboratori Nazionali di Frascati, Italy

October 9-14, 2011

\footnotetext{
1 Speaker
} 


\section{Introduction}

An electric dipole moment (EDM) aligned with a particle's spin violates both parity conservation and time reversal invariance. Any EDM discovery at present or proposed sensitivity levels would indicate a new physical process, one that may be needed to explain the surplus of matter over antimatter in the universe. Detection methods rest mainly on observing the precession of the spin in an external electric field. A storage ring provides an inward, radial electric field in the particle frame and, if an EDM exists, the possibility to observe it through a measurement of the rotation of the beam polarization from the longitudinal into the vertical direction. ${ }^{1}$ Care must be taken with the choice of electric and/or magnetic ring fields to suppress precession of the polarization in the ring plane. ${ }^{1}$

Developments are underway at the Cooler Synchrotron (COSY) located at the Forschungszentrum Jülich to demonstrate the techniques needed for this project. In order to achieve a sensitivity of $10^{-29} \mathrm{e} \cdot \mathrm{cm}$ with a maximum of $2 \times 10^{11}$ particles stored in the ring, the beam polarization measurement must detect a change in the vertical polarization component as small as $4 \times 10^{-6}$ during a storage time of about $1000 \mathrm{~s}$. For this time, the particle spins should not significantly decohere due to the spin tune differences that accompany a finite emittance or momentum spread. This requirement demands a polarimeter that continuously samples the beam and records the polarization with high efficiency: for every 100 particles extracted from the beam, one should be scattered from a suitable target material and recorded for the polarization calculation. The sensitivity to polarization should be high: the analyzing power should exceed 0.5. In addition, means must exist to correct any systematic errors arising from geometric or counting rate changes during the beam store. These requirements were met in a first study ${ }^{2}$ in 2009 with polarized deuterons at COSY that is summarized below. The work to investigate how to increase the spin coherence lifetime started in 2011 and initial results for an electron-cooled beam are given. Further work on higher order field corrections is planned.

\section{Polarimeter data analysis and systematic error correction}

Medium energy elastic scattering is particularly sensitive to beam polarization because of the large spin-orbit interaction with the nucleus. Carbon is often the preferred target. For a polarization component perpendicular to the scattering plane, a vector polarization produces a left-right counting rate asymmetry. Many contributions that are first-order in the systematic errors are canceled if both left and right counting rates are measured, data is available for positive and negative polarization states, and the information is combined in a cross ratio

$$
\varepsilon=\frac{3}{2} p A_{Y}=\frac{r-1}{r+1} \quad \text { where } \quad r^{2}=\frac{C(L,+) C(R,-)}{C(L,-) C(R,+)}
$$

and $p$ is the polarization, $A_{\mathrm{Y}}$ is the analyzing power, and the $C(i)$ are the counting rates.

Figure 1 shows measurements of $\varepsilon$ made with the In-Beam Polarimeter at the KVIGroningen by moving the beam off axis on a carbon target. A clear quadratic dependence is 
evident along with a linear error whose magnitude also depends on the difference between the magnitudes of the positive and negative polarizations.

Such higher-order errors can be corrected if the sensitivity of the polarimeter system to geometric and rate changes is calibrated to the $10^{-6}$ level and there is a means to estimate the size of these systematic effects. This study examined the use of two driving terms, one for geometry

$$
\phi=\frac{s-1}{s+1} \text { where } s^{2}=\frac{C(L,+) C(L,-)}{C(R,+) C(R,-)}
$$

and another for rate

$$
W=C(L,+)+C(R,+)+C(L,-)+C(R,-)
$$

to be added to the cross ratio formula to produce

$$
\varepsilon=\frac{r-1}{r+1}-\left(\frac{\partial \varepsilon}{\partial \phi}\right) \Delta \phi-\left(\frac{\partial \varepsilon}{\partial W}\right) \Delta W .
$$

The derivatives in Eq. (4) may in general be functions of $\phi$ and $W$, thus permitting correction for effects beyond second-order.

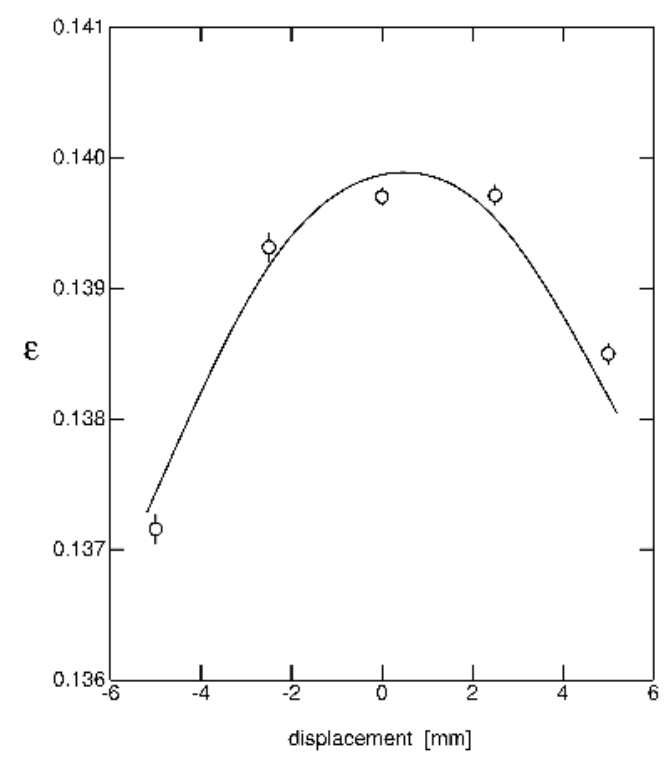

Figure 1. Asymmetry ( $\varepsilon$ ) data for $d+C$ scattering at $\theta_{\mathrm{LAB}}=18^{\circ}$ and $110 \mathrm{MeV}$ as a function of the beam displacement on target. The curve is based on systematic error derivatives for Eq. (4) obtained from elastic scattering angular distributions.

\section{Polarimeter efficiency and analyzing power at COSY}

Running at COSY made use of the EDDA scintillation detectors as a mock EDM polarimeter operating between $\theta_{\mathrm{LAB}}=9.0^{\circ}$ and $13.2^{\circ}$. The beam momentum of $p=0.97 \mathrm{GeV} / c$ was chosen to emphasize signals from elastically scattered deuterons that stopped in the outer scintillators. The beam was slowly extracted by using white noise applied to a pair of vertical electric field plates. Extracted beam struck the leading face of a carbon block mounted above the beam. Its $15-\mathrm{mm}$ thickness made possible a polarimeter efficiency of $0.7 \times 10^{-3}$, a value consistent with the desired $1 \%$ if a $5-\mathrm{cm}$ target were to be used and scattering observed at angles down to $5^{\circ}$ (based on Monte Carlo simulations). The effective analyzing power was $3 A_{\mathrm{Y}} / 2=$ $0.67(4)$ with the error dominated by the calibration of the beam polarization.

\section{Systematic error correction at COSY}

Asymmetry measurements were made with the EDDA mock polarimeter for a variety of deliberately induced geometry errors, including position displacements as large as $2 \mathrm{~mm}$ and angle changes as large as $5 \mathrm{mrad}$. Four polarization states with different vector and tensor polarization values were used along with an unpolarized state. In addition to calculating the cross ratio, simpler asymmetries such as $\varepsilon=(L-R) /(L+R)$ and the tensor-sensitive combination comparing horizontal and vertical plane scattering were examined. The data were also combined to yield the geometric and rate driving terms given by Eqs. (2) and (3). 


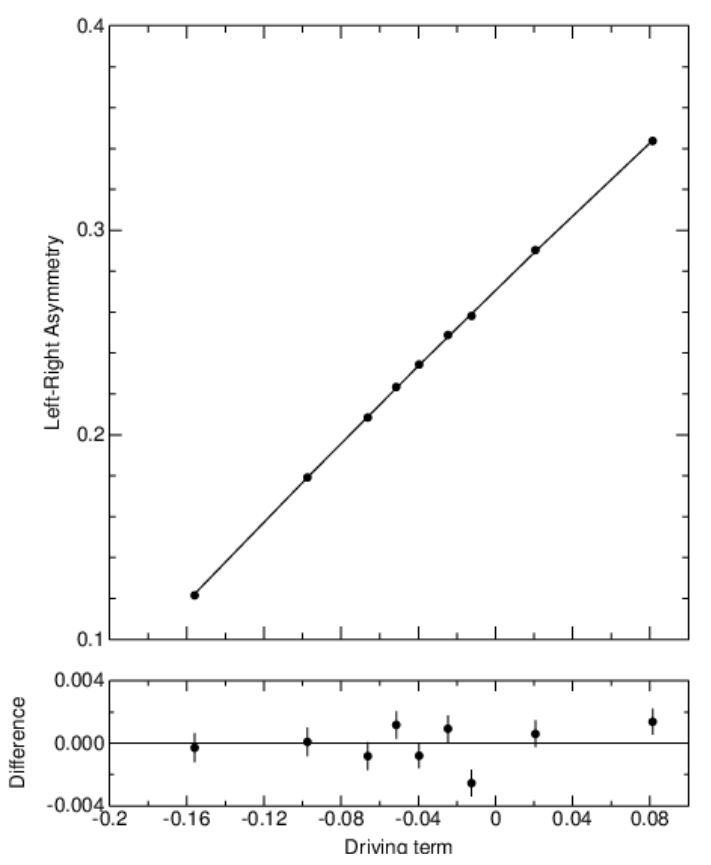

Figure 2. Left-right asymmetry data as a function of the geometric driving term $\phi$. The curve is a model determination of the correction function, subtracted in the lower panel to illustrate the statistical errors.

Figure 2 shows the left-right asymmetry for a combined vector and tensor polarized state as a function of the geometric driving term. (The curve is from a model discussed in the next section.) The five closely spaced points in the middle represent five beam positions; the more widely spaced points five beam angles. All of these data are consistent with a single correction curve, the values of the geometric derivative in Eq. (4) as a function of $\phi$. If this property of the systematic error calibration holds, then it becomes possible to make all geometric corrections using only $\phi$. No additional information is needed, and the correction becomes possible in real time with only the primary count rates as input.

Figure 3 shows an example of how this correction works during the time of a beam store. A left-right asymmetry is shown again because the systematic error effects are enhanced. The red points are the original asymmetry measurements made as a function of time. The asymmetry rises briefly, then falls for the rest of the store. This is a rate effect whose sign is consistent with rates increased by pileup in the photomultiplier signals. In the COSY study, all such effects were linear in rate and thus corrected with a single coefficient. Removing only the rate term yields the blue points which now are constant with time. But there is also a significant geometric error. Correcting for this produces the black points. The straight line through these points has a slope of $[-4$ $\pm 11] \times 10^{-6}$, indicating no polarization change within the statistical limit of this test. Additional tests with time-dependent geometrical errors gave similarly good corrections.

Corrections to the cross ratio asymmetry are much smaller than those

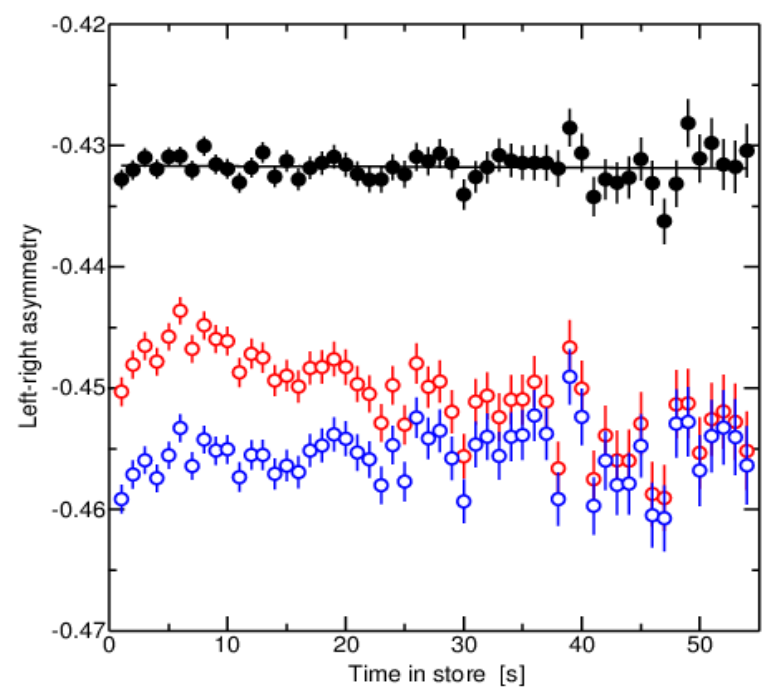

Figure 3. Left-right asymmetry measurement across a beam store. The red (no correction), blue (rate), and black (rate and geometry) points show the progressive improvements from systematic error corrections. shown in Figs. 2 and 3. When combined with the smaller geometry and angle changes expected during the operation of the EDM experiment, the corrections, and consequently the errors in the corrections, are both much smaller than the required $10^{-6}$. 


\section{Systematic error model}

To confirm that geometrical systematic errors were understood, a model was created in which position or angle errors were connected to specific effects through different mechanisms. These were added to the main effect, the changes in count rate created by the beam polarization and described by the analyzing power of the polarimeter's nuclear reactions. Each mechanism was parametrized with values determined by a fit to the asymmetry data. First, position and angle changes were related through the effective distance to the detector. Next changes to the cross section and analyzing power were represented by a Taylor series. Solid angle differences were included. Beyond these expected mechanisms, it was necessary to add the mixing of horizontal beam motion with vertical, a rotation of the EDDA detector, and a background that appeared on the low-momentum side of the beam. With these mechanisms in place, all systematic features of the data were reproduced.

The model reproduction of left-right and tensor asymmetries are shown in Figs. 4 and 5.

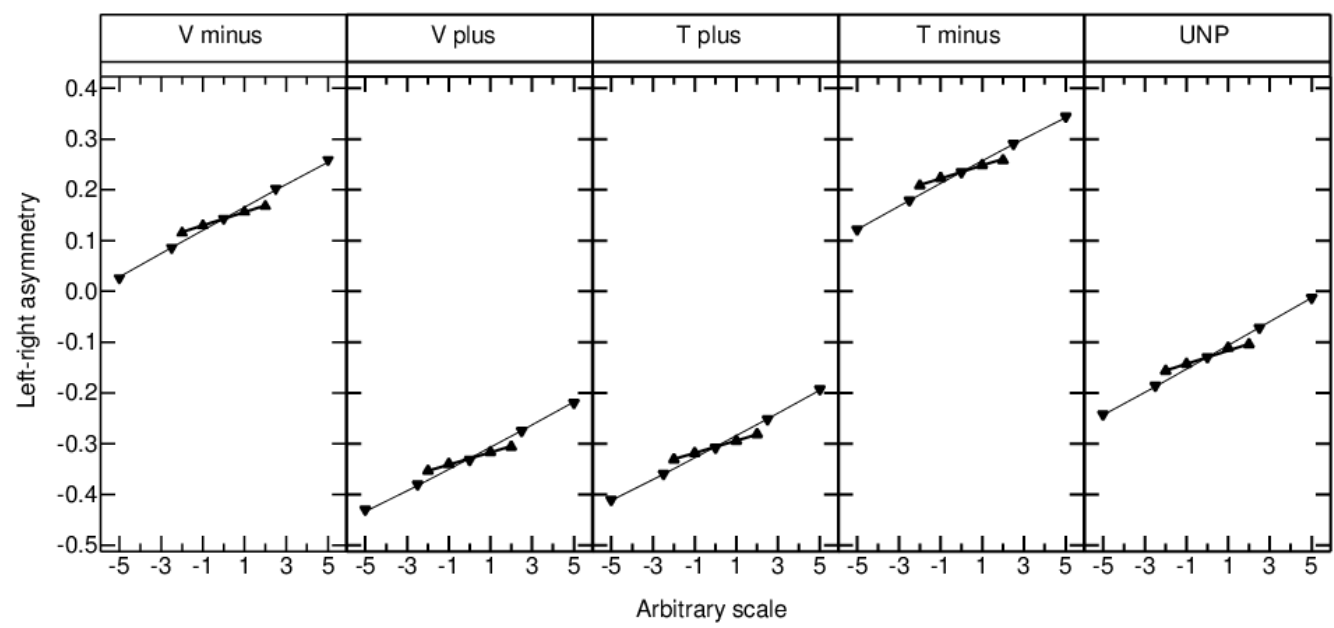

Figure 4. Left-right asymmetry measurement versus either position (horizontal scale in $\mathrm{mm}, \mathbf{\Delta}$ ) or angle (horizontal scale in mrad, $\mathbf{\nabla}$ ). The lines are error model calculations.

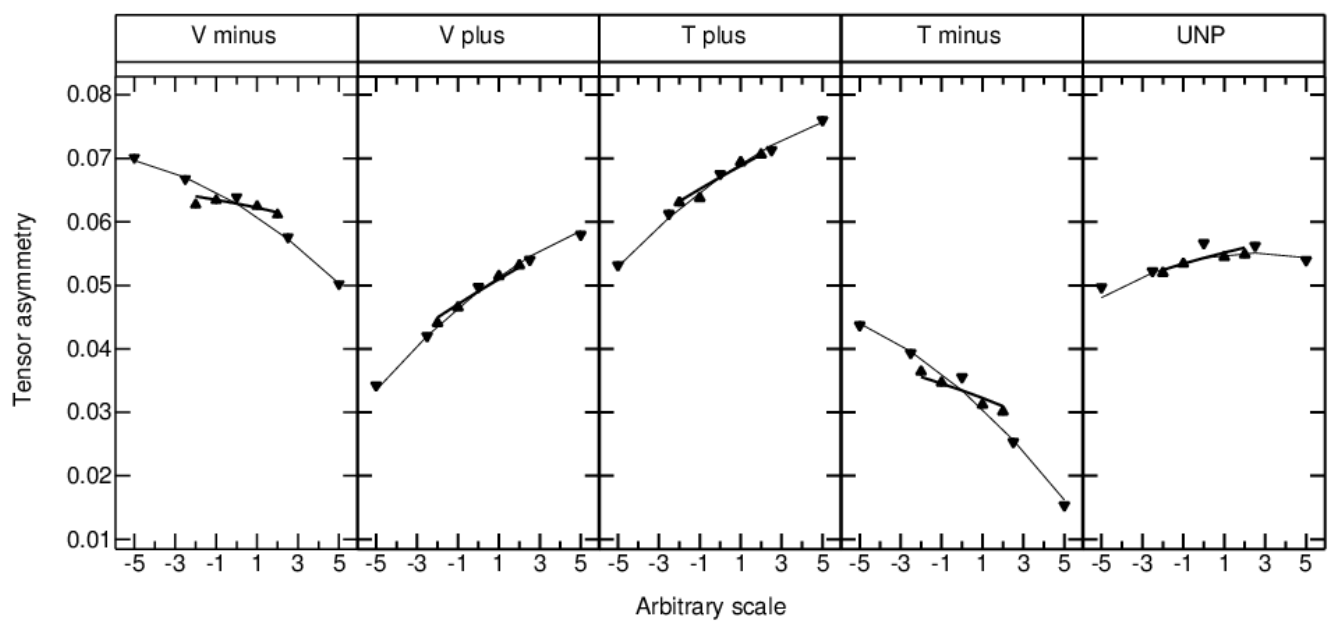

Figure 5. Tensor asymmetry measurement as in Fig. 4. 
The slopes in Fig. 4 reflect primarily the derivative of the cross section with either position or angle, which are similar for all polarization states. Small changes from one state to another make it possible to extract the derivative of the analyzing power. There is some tensor polarization in Fig. 5 for all of the polarized beam states. The slopes are the product of the vector asymmetry and the derivative of the cross section. The curvature determines the cross section second derivative. Other parameters were determined from additional asymmetry data. Occasional points (e.g., at 0 in Fig. 5 for T- and UNP states) suffered non-statistical operational problems, raising the reduced chi square for the model fit to all asymmetry data to 1.7.

\section{Investigation of spin coherence time}

The EDM signal is the rotation of the polarization from its initial direction parallel to the velocity into the vertical direction. Only the vertical polarization component is stable in a storage ring (without a Siberian Snake). Without either an all-electric ring (and the right momentum) in the proton case or crossed electric and magnetic fields in the deuteron case, the polarization will precess in the horizontal or ring plane at a high rate determined by the spin tune (relativistic parameter $\gamma$ multiplied by the anomalous magnetic moment). The momentum spread of the beam will quickly cause the longitudinal polarization to decohere. This first-order problem is removed by bunching the beam, thus forcing all orbit periods to be the same on average. However, emittance and small non-linear momentum dependences still lead to longer path lengths, changes to $\gamma$, and a spread in spin tunes. The feasibility of the EDM search requires a demonstration that corrections can lengthen the spin coherence time to about $1000 \mathrm{~s}$.

Strategies to reduce decoherence include beam cooling (either electron-beam or stochastic) to reduce emittance and momentum spread and correcting increased orbit lengths through the use of sextupole or higher order fields. COSY studies begun in January 2011 involved the use of an RF solenoid to manipulate the beam polarization. Measurements of the time evolution of the polarization with the RF solenoid running on or near the $1-G \gamma$ harmonic of the deuteron spin tune were expected to yield information on the RF solenoid resonance width, which also depends on emittance and momentum spread. In practice, comparisons between cooled and uncooled beams were complicated by the effects of changing synchrotron amplitude on the effective RF solenoid strength, as discussed by Guidoboni at this conference. ${ }^{3}$

\section{Tests of cooled beam spin coherence time}

Running the RF solenoid at its maximum strength $\left(\varepsilon=4.05 \times 10^{-6} \mathrm{rev}\right)$ at a frequency as close as feasible to the resonant frequency $(<0.1 \mathrm{~Hz})$ produced a long-lived modulation of the vertical polarization with no consistent deterioration in its magnitude when the process was started with electron-cooled beam. Such behavior suggests that the spins in the beam remain coherent for a long time. By ramping the solenoid power down, it proved possible to stop this modulation at a point where the vertical polarization was close to zero. At this place, the polarization was in the horizontal plane and rotating at $\mathrm{G} \gamma \mathrm{f}_{\mathrm{CYC}}=120 \mathrm{kHz}$. If the solenoid was turned on again after some delay, it was possible to recreate the modulation pattern. This required that the solenoid catch the rotating polarization at the correct phase and do so 
reproducibly on subsequent stores so that the result could be accumulated for best statistics. Such reproducibility was not possible for delay times longer than about $30 \mathrm{~s}$.

One example of such a measurement is shown in Fig. 6 where the solenoid is off for $25 \mathrm{~s}$. The vector polarization information has been averaged for all polarization states and normalized to one before the solenoid is first turned on. During the periods of modulation, lines connect consecutive data points to aid the eye. Most of the polarization is recovered, which provides a lower limit on the spin coherence time of $25 \mathrm{~s}$. Any polarization losses cannot be distinguished from problems with the solenoid phase or the stability of the control system.

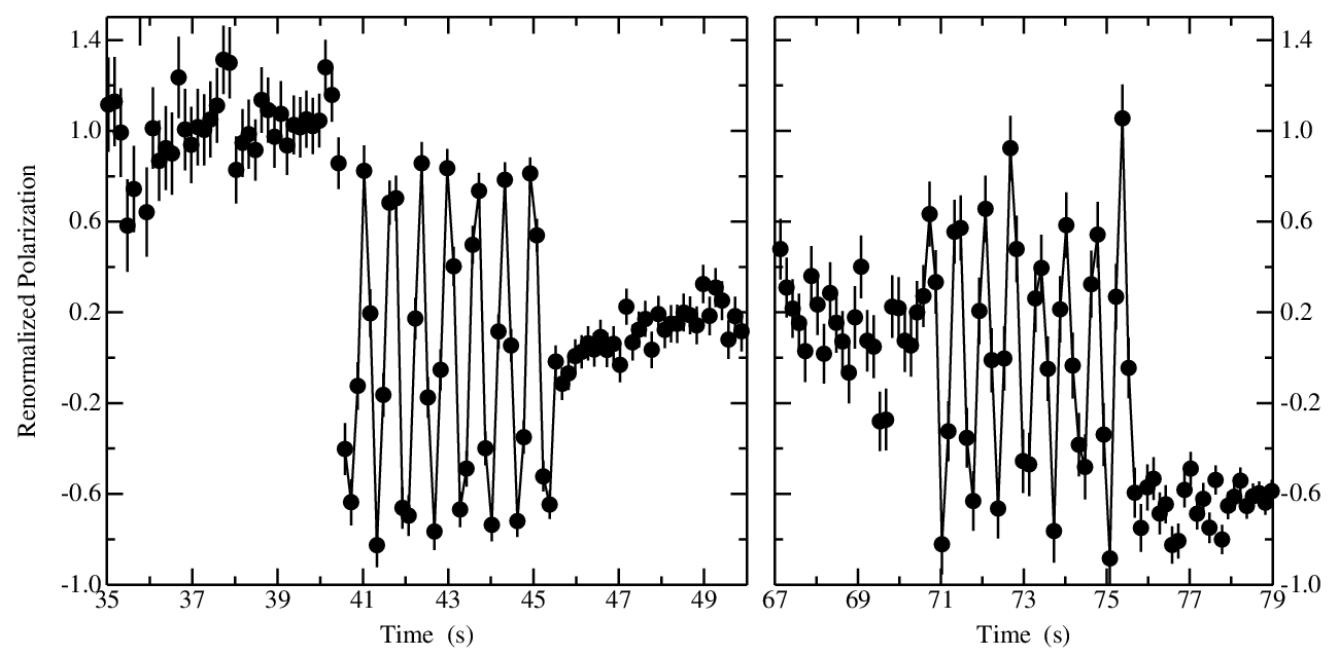

Figure 6. Renormalized vertical polarization measurements as a function of time during a store. The RF solenoid was on for $5 \mathrm{~s}$ between 40.5 and $45.5 \mathrm{~s}$, and again between 70.5 and $75.5 \mathrm{~s}$. Power ramp times were $0.2 \mathrm{~s}$. The lines connect consecutive data points to guide the eye.

Tests as shown in Fig. 6 were made for several delay times. The tests with the largest polarization recovery are shown in Fig. 7. In parallel with these tests, measurements were made of the beam emittance by observing the beam profile and comparing it with transport predictions. The emittance was then simulated by a Gaussian distribution of betatron oscillation amplitudes and the decoherence of the ensemble calculated after tracking the evolution of the individual particle spins in time. The blue band represents this prediction for the recovered polarization where the width of the band represents the Monte Carlo error. All of the measurements fall below the band, as

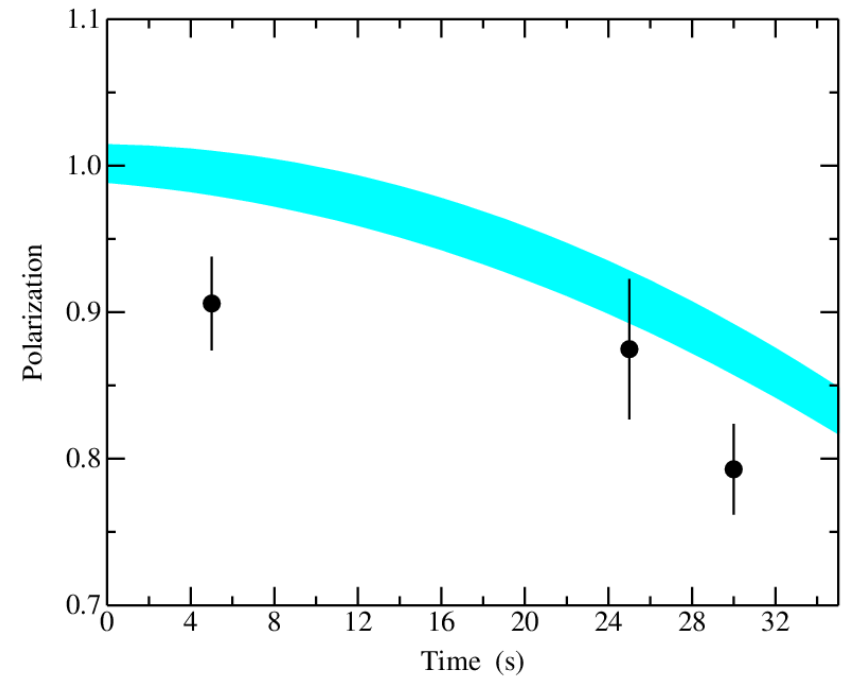

Figure 7. Lower limits on the recovered polarization as a function of the delay before restarting the solenoid. The blue band is a Monte Carlo estimate of the emittance contribution to depolarization. Its width is the Monte Carlo error. 
expected for lower limits. If the emittance is increased to make the band pass through the data point at $30 \mathrm{~s}$, half of the polarization would be lost after $75 \mathrm{~s}$, the shortest horizontal polarization lifetime consistent with these lower limits.

Analysis of a variety of measurements with both cooled and uncooled beam is underway. ${ }^{3}$ From these data, it is possible to deduce the longitudinal distribution of the uncooled beam. For planning the next phase of this development project, tracking simulations have been made to determine the best procedure for producing a high degree of horizontal polarization under a variety of beam conditions. This appears to be a Froissart-Stora frequency scan that is stopped on or close to the resonance. It is planned to use time-stamped data to unfold the rotating polarization for a direct measurement of the horizontal polarization components and their lifetime. This will be used to track the effects of changing emittance and momentum spread as well as the efficacy of various means to lengthen the coherence time.

\section{Summary}

Using existing detectors at the KVI and COSY, it has been demonstrated that second order geometric errors limit the ability to observe small polarization changes in a storage ring EDM search, but these become correctable in real time if error driving terms are calculated and a calibration is made of the polarimeter sensitivity to such errors. By using slow extraction into a thick target close to the beam, high polarimeter efficiencies are possible while retaining a large sensitivity to the polarization. New studies are underway of the spin coherence time. Still needed is an effort to extend the existing lower limit about an order of magnitude to reach the EDM search goals.

The collaborators wish to thank the US NSF, Innovational Research and Toptalent grants from the Dutch government, the Italian INFN, and the Helmholtz Foundation and the EU for their support of this work at the KVI and COSY.

Additional collaborators on this work include N.P.M. Brantjes, D.J. van der Hoek, W. Kruithof, M. da Silva e Silva, and O.O. Versolato (KVI); D. Chiladze, J. Dietrich, M. Gaisser, R. Gebel, V. Hejny, A. Kacharava, A. Lehrach, B. Lorentz, R. Maier, D. Mchedlishvili, A. Polyanskiy, D. Prasuhn, F. Rathmann, H. Stockhorst, H. Ströher, Yu. Valdau, Ch. Wiedeman, and P. Wüstner (COSY-Jülich); V. Dzordzhadze, D. Lazarus, W.M. Morse, and Y.K. Semertzidis (BNL); F.E. Gray (Regis); P. Levi Sandri and G. Venanzoni (Francati); F. Gonnella, R. Messi, and D. Moricciani (U. Rome), P. Benati, G. Guidoboni, and A. Pesce (Ferrara); P. Kulessa (Jagiellonian U.); and R. Talman (Cornell).

\section{References}

[1] F.J.M. Farley et al., New Method of Measuring Electric Dipole Moments in Storage Rings, PRL 93 (2004) 052001.

[2] N.P.M. Brantjes et al., Correcting systematic errors in high-sensitivity deuteron polarization measurements, NIM A 664 (2012) 49.

[3] G. Guidoboni, Synchrotron Oscillation Effects on Observations of an RF-solenoid Spin Resonance for a Polarized Deuteron Beam at COSY, in proceedings of STORI'11 conference POS (STORI11) 062. 